\title{
ANÁLISE DO PERFIL QUÍMICO DO LEITE HUMANO DE MÃES USUÁRIAS DO CRACK
}

Fernanda Braghini ${ }^{1}$, Vanessa Jaqueline Baptista ${ }^{1}$, Mayara Marques Mota $^{2}$, Cintia Farinazo $^{2}$, Jose Eduardo Gonçalves ${ }^{1,3}$

${ }^{1}$ Programa de Pós-graduação em Promoção da Saúde, Centro Universitário de Maringá - UniCesumar - Maringá - PR. Brasil. (fernanda.braghini@hotmail.com)

${ }^{2}$ Centro Universitário de Maringá - UniCesumar - Maringá - PR, Brasil.

${ }^{4}$ Instituo Cesumar de Ciências, Tecnologia e Inovação - ICETI

Recebido em: 03/10/2016 - Aprovado em: 21/11/2016 - Publicado em: 05/12/2016

DOI: 10.18677/EnciBio 2016B 127

O leite é a primeira alimentação humana e fonte de nutrientes para as funções biológicas, sendo considerado o melhor alimento para criança nos primeiros seis meses de vida. É de extrema importância que a mãe evite o consumo de drogas lícitas e ilícitas que podem prejudicar a qualidade do leite humano (LH). O objetivo deste trabalho consiste na análise do perfil químico do LH em mães usuárias do crack na determinação de proteínas, açúcares totais, gorduras e minerais. Para o estudo utilizou-se LH de duas mães usuárias de crack, sendo analisados Acidez Dornic, Crematócrito, Proteínas, Açúcares, Gorduras, Minerais e Lactose. Os dados obtidos foram analisados e os resultados encontrados para cada metodologia correspondem aos valores médios para o LH das duas mães: Acidez Dornic 26,50 D \pm 4,97; Crematócrito: Teor de Creme 7,63 \pm 5,10 \%, Gordura 4,82 $\pm 3,49 \%$, Valor Energético de 799,7 $\pm 341 \mathrm{Kcal} / \mathrm{L}$; Proteínas totais $12,90 \mathrm{~g} / \mathrm{L} \pm 0,28$; Glicose 37,06 \pm $0,83 \mathrm{mg} / \mathrm{dL}$; Sacarose 11,07 \pm 0,25 g/dL; Lactose 20,31 \pm 7,90 g/dL; Minerais, Na $25,4 \pm 5,89 \mathrm{mg} / \mathrm{dL}, \mathrm{K} 7,5 \pm 2,34 \mathrm{mg} / \mathrm{dL}$ e $\mathrm{Ca} 23,1 \pm 1,75 \mathrm{mg} / \mathrm{dL}$. Estes resultados demostraram que o LH de mães usuárias do crack, apresentou alterações nas concentrações de proteína, glicose, sacarose e o mineral potássio, onde encontramse abaixo do estabelecido e para o sódio, cálcio e lactose os valores estão acima dos estipulados em literatura. Conclui-se que o leite das mães usuárias do crack, apresentam composição nutricional diferente do LH de mães não usuárias do crack.

PALAVRAS-CHAVE: Composição nutricional; Leite materno; Promoção da Saúde.

\section{ANALYSIS PROFILE CHEMIST BREASTFEEDING MOTHERS OF USERS OF CRACK MILK BREAST MILK CHEMICAL ANALYSIS OF ADDICTED MOTHERS.}

\begin{abstract}
Considering the importance of breastfeeding, milk is the first food and nutrient source for the biological functions and is considered the best food for children in the first six months of life. Therefore, during this process, it is extremely important that the mother avoid the consumption of licit and illicit drugs that can impair the quality of breast milk. Therefore the aim of this study is to analyze the chemical profile of breast milk of mothers who crack the determination of protein, total sugars, fats and minerals through specific chemical methods for each constituent. For the study we ENCICLOPÉDIA BIOSFERA, Centro Científico Conhecer - Goiânia, v.13 n.24; p. 13752016
\end{abstract}


used human milk two crack mothers who met at the University Hospital of MaringáPR. analyzes Acidity Dornic were applied, creamatocrit, Total Protein, Total Sugars, fats, minerals, lactose and Total Acidity. The data were analyzed and according to the average of these the results for each methodology: Acidity Dornic $66.50{ }^{\circ} \mathrm{D} \pm$ 19.09; Total Protein $12.90 \mathrm{~g} / \mathrm{L} \pm 0.28$; Glucose $0.37056 \mathrm{mg} / \mathrm{dL} \pm 0,8344 \times 10-2$; Sucrose $0.11065 \mathrm{mg} / \mathrm{dL} \pm 2,47 \times 10-2$; Fats $2,20 \mathrm{~g} / \mathrm{dL} \pm 0.47$; Lactose $20.31 \mathrm{~g} / \mathrm{dL} \pm$ 7.90; Total Acidity $(\mathrm{pH}) 2.58 \pm 0.042$; Minerals Sodium 58,37mg / dl, Potassium $29,33 \mathrm{mg} / \mathrm{dL}$ Calcium and 46,2mg / dL; Creamatocrit: $7.63 \%$ cream $\pm 5.10 ; 4.82 \%$ fat $\pm 3.49 ; 799,7 \mathrm{kcal} / \mathrm{L} \pm 341$. Thus it can be concluded that the milk of mothers who crack, showed considerable changes in the concentrations of the components analyzed when compared to the studies of mothers do not crack users.

KEYWORDS: Breast milk; Chemical composition; Crack.

\section{INTRODUÇÃO}

A amamentação é reconhecida mundialmente como a forma mais apropriada de fornecer uma alimentação saudável para o recém-nascido, de maneira exclusiva nos primeiros seis meses de vida, podendo estender-se de maneira complementar até os dois anos ou mais. (LEVY \& BÉRTOLO, 2012).

Essa importância se dá ao fato do leite ser uma fonte rica de nutrientes para as funções biológicas do recém-nascido, sendo constituído basicamente de proteínas, açucares, gorduras, minerais e vitaminas (CALIL \& FALCÃO, 2003; CURY, 2003), além de exercer um papel muito importante na proteção imunológica contra doenças infecciosas, na adequação nutricional e no desenvolvimento afetivo e psicológico (MORGANO et al., 2005). Portanto, nenhum fator externo que pode prejudicar a qualidade do leite materno e a saúde da criança pode interferir neste processo, como por exemplo, o uso de drogas lícitas e ilícitas.

O crescimento do consumo do crack no Brasil tornou-se um fenômeno de saúde pública, sendo hoje chamado de epidemia do crack. É um derivado da cocaína, um estimulante do sistema nervoso central, podendo ser injetado ou aspirado sob a forma de sal hidrossolúvel, o cloridrato de cocaína, ou ser convertido em sua forma alcalina, a pasta básica, ou o crack (PULCHERIO et al., 2010).

Sabe-se que o uso de crack durante a gestação pode desencadear abortos espontâneos, prematuridade, diminuição no crescimento do feto, outras alterações perinatais e até sintomas relacionados à intoxicação e/ou abstinência após o parto pelo recém-nascido (CROUCH 1993; CASATTI, 2011; GASPARIN et al., 2012), porém, quando correlacionado a amamentação, acredita-se que a sua presença está diretamente relacionada à diminuição no tempo de amamentação, devido à baixa produção do hormônio prolactina e a possível presença do crack ou dos derivados metabólitos no leite humano (AMERICAN, 2001), porém pouco se sabe sobre seus prejuízos no perfil nutricional do leite humano.

O interesse pelo estudo e análise do leite materno é baseado em vários fatores como: variações da composição, a importância nutricional e funcional deste alimento (VELOSO et al., 2001; SALDIVA, 2011). Para isso, são necessários métodos que permitam avaliar e quantificar os elementos que o compõe (SILVA et al., 2003). As concentrações de proteínas, açúcares totais e de gorduras podem ser analisadas por diferentes métodos, tais como por fixação de corante, espectrofotométricos por absorção no infravermelho e absorção no ultravioleta; cromatografia líquida, eletroforéticos, entre outros (VELOSO et al., 2001).

Portanto, visando identificar os prejuízos que o crack causa da qualidade do leite materno, este estudo tem como objetivo quantificar a presença de proteínas, 
açúcares totais, gorduras e minerais presentes no leite materno de mães dependentes químicas do crack, a fim de comparar seu valor nutricional com o leite materno de mães não usuárias.

\section{MATERIAL E MÉTODOS}

O estudo realizado trata-se de uma pesquisa quantitativa transversal e de coorte, desenvolvida no município de Maringá/PR no ano de 2015, através da análise química do leite humano $(\mathrm{LH})$ de amostras obtidas de duas mães usuárias de crack atendidas pelo Hospital Universitário (HU) da Universidade Estadual de Maringá - UEM. As amostras de LH foram coletadas e armazenadas pelo banco de leite humano do HU e cedidas para o Laboratório de Análises Químicas e Biológicas do UniCesumar, onde foram desenvolvido todos os procedimentos para a quantificação da composição química deste LH.

\section{Acidez Dornic}

Para a coleta utilizou-se tubo de ensaio $5 \mathrm{~mL}$ da amostra de LH em campo de chama ( $1 \mathrm{~mL}$ de cada vez e coletar em diferentes campos da amostra), logo apos a amostra foi conservada refrigerada (abaixo $5^{\circ} \mathrm{C}$ ) e $\mathrm{m}$ banho de gelo por 30 minutos. Após 30 minutos a amostra foi retirada do banho de gelo, agitada em vórtex e uma alíquota de um $\mathrm{mL}$ foi transferida para outro tubo. Esse procedimento foi repetido duas vezes. Em cada tubo foi adicionada uma gota de solução hidroalcólica de fenolftaleína e a amostra de LH titulada com solução padrão de Hidróxido de Sódio $0,01 \mathrm{~mol} / \mathrm{L}$. Na análise cada $0,01 \mathrm{ml}$ gasto de solução padrão de $\mathrm{NaOH}$ correspondeu a 19D (São Paulo, 2008).

\section{Crematócrito}

O teor de creme que também é conhecido como técnica do crematócrito foi realizado conforme o método de LUCAS Et. al. (1978), adaptado de FLEET \& LINZELL (1964).

\section{Proteínas Totais}

O método que utilizado para a determinação da concentração protéica do leite materno foi o de Bradford o qual utiliza o reagente Comassie-Blue. Para o preparo desse reagente foi pesado $30 \mathrm{mg}$ do corante Comassie-Blue brilhant dissolvido em $15 \mathrm{~mL}$ de Etanol 95\% e $30 \mathrm{~mL}$ de Ácido Fosfórico 85\%, em seguida diluído com água destilada até o volume de $300 \mathrm{~mL}$. Realizou-se 3 filtrações do reagente até que conseguiu-se chegar a uma absorbância de aproximadamente 0,2 em $595 \mathrm{~nm}$.

Para quantificação da proteína do LH preparou-se uma solução padrão de Albumina de Soro Bovino (ASB) $0,25 \mathrm{mg} / \mathrm{mL}$. À partir desta solução foi estabelecida a curva de calibração variando concentração de 0,5 à $5 \mu \mathrm{g} / \mathrm{mL}$ ASB, onde para cada concentração do padrão foi adicionado $5 \mathrm{~mL}$ do reagente Comassie-Blue brilhante.

Para a leitura das amostras de $\mathrm{LH}$, foi diluído $0,5 \mathrm{~mL}$ de amostra em $9,5 \mathrm{~mL}$ de água destilada, $1 \mathrm{~mL}$ do LH diluído foi transferido para outro tubo e acrescentado $5 \mathrm{~mL}$ do Reagente Comassie-Blue brilhante. A absorbância da amostra e dos padrões foram medidas em espectrofotômetro Varian Cary 100 1E, utilizando lâmpada de tungstênio, a um comprimento de onda de 595 nm contra o "branco" $100 \mu \mathrm{L}$ de água destilada em $5 \mathrm{~mL}$ de reagente Comassie-Blue brilhante. $\mathrm{O}$ procedimento foi realizado em triplicata para cada amostra. 


\section{Açúcar Total}

A análise de açúcar total foi realizada utilizando o Reagente de Fenol Sulfúrico. O método constituiu-se no preparo de uma solução padrão de glicose $2.500 \mu \mathrm{g} / \mathrm{mL}$, identificada como P1 e uma segunda solução padrão de glicose preparada a partir da diluição da solução P1 para obter a solução padrão de glicose $250 \mu \mathrm{g} / \mathrm{mL}(\mathrm{P} 2)$.

A curva de calibração padrão de glicose foi preparada na razão de concentração entre 10 à $55 \mu \mathrm{g} / \mathrm{mL}$ a partir da solução P2. Já as amostras de LH foram preparadas diluindo $0,5 \mathrm{~mL}$ em $9,5 \mathrm{~mL}$ de água destilada. Para as medidas espectrofotométricas, a $1 \mathrm{~mL}$ (amostra ou padrão de glicose) foram adicionados 0,5 $\mathrm{mL}$ de fenol a $5 \%$ e $2,5 \mathrm{~mL}$ de $\mathrm{H}_{2} \mathrm{SO}_{4}$ concentrado, agitado e repouso por $10 \mathrm{~min}$. Em seguida banho-maria a $25{ }^{\circ} \mathrm{C}$ por $10 \mathrm{~min}$. A leitura efetuado em um espectrofotômetro Varin Cary 100 1E em 490 nm.O mesmo procedimento foi realizado para fazer a curva padrão de sacarose.

\section{Minerais}

Os minerais $\mathrm{Ca}^{+2}, \mathrm{~K}^{+}$e $\mathrm{Na}^{+}$foram analisados em fotômetro de chama (Analyser 910), e para isso foi necessária a preparação de soluções padrões contendo $140 \mathrm{ppm}$ de $\mathrm{Na}^{+}, 5 \mathrm{ppm}$ de $\mathrm{K}^{+}$e $\mathrm{Ca}^{2+} 30 \mathrm{ppm}$ dos respectivos íons. Para as amostras, foram diluídos $0,5 \mathrm{~mL}$ de cada LH em um balão volumétrico de $100 \mathrm{~mL}$ e as leituras foram efetuada no fotômetro de chama após a calibração do 100 \% com as soluções padrões.

\section{Lactose}

\section{Determinação de glicídios redutores em lactose}

Procedimento - Transferiu-se, com auxílio de uma pipeta volumétrica, $10 \mathrm{~mL}$ da amostra para um balão volumétrico de $100 \mathrm{~mL}$, e foram adicionados $50 \mathrm{~mL}$ de água, $2 \mathrm{~mL}$ da solução de sulfato de zinco a $30 \%$ e $2 \mathrm{~mL}$ da solução de ferrocianeto de potássio a $15 \%$, misturando bem após cada adição. Deixou-se sedimentar durante 5 minutos, completou-se volume com água, agitou-se e filtrou. Em um balão de fundo chato de $300 \mathrm{~mL}$, foram adicionados $10 \mathrm{~mL}$ de cada uma das soluções de Fehling e $40 \mathrm{~mL}$ de água, aquecendo até a ebulição. O filtrado foi transferido para uma bureta de $25 \mathrm{~mL}$ e adicionado sobre a solução do balão em ebulição com agitação vigorosa, até que esta solução mudasse de coloração azul à incolor (no fundo do balão ficou um resíduo vermelho-tijolo).

\section{RESULTADOS E DISCUSSÃO}

$\mathrm{O}$ LH é uma mistura homogênea rica em micro e macro nutrientes que além de fornecer hormônios, aminoácidos essenciais e fatores imunológicos atende as necessidades nutritivas, metabólicas e digestivas do RN (VIEIRA et al., 2004). Apesar das inúmeras vantagens, a concentração de nutrientes sofre variações ao longo do dia e também ao longo do período de amamentação, principalmente nas concentrações de proteínas, carboidratos, lipídeos e eletrólitos (STEVANATO et al., 2012).

As amostras de LH de duas mães dependentes químicas (Crack) foram coletas e armazenadas pelo banco de leite do Hospital Universitário da Universidade Estadual de Maringá e cedidas para análise da composição química, uma vez que estas amostras não poderiam ser utilizadas para o consumo humano. 
$\mathrm{Na}$ análise de acidez (Tabela 1) os resultados mostram uma acidez muito elevada para as duas amostras de $\mathrm{LH}$, caracterizando-as como fora dos padrões com acidez titulável muito superior a $7^{\circ} \mathrm{D}$.

TABELA 1: Acidez Titulável e total para o LH

\begin{tabular}{lcc}
\hline & $\mathrm{LH}_{1}$ & $\mathrm{LH}_{2}$ \\
\hline Acidez Dornic $\left({ }^{\circ} \mathrm{D}\right)$ & $23 \mathrm{D}$ & $30 \mathrm{D}$ \\
Acidez total $(\%)$ & 0,004 & 0,004 \\
pH & 2,55 & 2,61 \\
\hline
\end{tabular}

A média de acidez Dornic encontrada nas duas amostras foi de $26,50 \pm 4,97$ D. Este dado demonstra uma acidez elevada, quando comparado com o estudo realizado por CAVALCANTE et al., (2005), mostrando assim que estas amostras não poderiam ser utilizadas como fonte de alimento dos recém-nascidos.

De acordo com o Ministério da saúde o leite materno deve apresentar uma acidez Dornic menor que 7D para poder ser consumido. CAVALCANTE et al., (2005)., citam que a acidez do leite pode aumentar conforme se diminui a temperatura de armazenamento do leite e que pode ser aumentada também de acordo com a concentração de lipídios, ou seja, quanto mais lipídios presentes maior é a acidez do leite.

A acidez total através do $\mathrm{pH}$ do leite foi expressa em porcentagem e apresentou média do $\mathrm{pH}$ obtido das amostras de LH de $2,58 \pm 0,042$. $\mathrm{O} \mathrm{pH}$ do leite humano ordenhado deve encontrar-se entre 5,47 à 7,84 , variando diretamente com as semanas de lactação e indiretamente com o percentual lipídico, sendo assim é coerente dizer que quanto maior a concentração de gordura total maior seria a possibilidade do leite desenvolver elevada acidez (CAVALCANTE et al., 2005; STEVANATO et al., 2012). Comparando os resultados obtidos de LH de mães dependentes química com o estabelecido, observa-se uma acidez aumentada no leite dessas mães, o que torna este leite impróprio.

O crematócrito (VIEIRA et al., 2004) é um micrométodo rápido de controle de qualidade do $\mathrm{LH}$, através do qual se determina a quantidade de creme e se estima a concentração de gordura e o conteúdo energético (SILVA et al., 2007) de uma amostra, como apresentado na Tabela 2.

TABELA 2: Teor de creme, gordura total estimada e valor energético para os LH de mães dependentes químicas (Crack).

\begin{tabular}{lcc}
\hline & $\mathrm{LH}_{1}$ & $\mathrm{LH}_{2}$ \\
\hline Teor de creme (\%) & 11,24 & 4,02 \\
Gordura total estimada (\%) & $7,29 \%$ & 2,35 \\
Valor energético (Kcal/L) & $1.040,83$ & 558,54 \\
\hline
\end{tabular}

Considerando-se que quanto menor é o pH e maior a acidez titulável, maior deve ser a concentração de lipídios presente no LH. Na análise do teor de creme médio encontrado foi de 7,63 $\pm 5,10 \%$ (Tabela 2), valor este de acordo com os valores encontrados para $\mathrm{pH}$ e acidez (Tabela 1). Estes valores são muitos superiores aos relatados por outros pesquisadores (VIEIRA et al., 2004; CAVALCANTE et al., 2005; STEVANATO et al., 2012).

Através do teor de creme foi possível estimar se a gordura média 4,82 \pm $3,49 \%$ e valor energético de 799,7 $\pm 341 \mathrm{Kcal} / \mathrm{L}$ (Tabela 2). De acordo com CAVALCANTE et al.,(2005), o leite humano que pode ser administrado para o recém ENCICLOPÉDIA BIOSFERA, Centro Científico Conhecer - Goiânia, v.13 n.24; p. 13792016 
nascido, deve conter uma distribuição de lipídios que varia de 1 a 24\%, 40g de gordura/L e em média $700 \mathrm{Kcal} / \mathrm{L}$, já segundo CURY (2009), o leite de colostro deve conter em média $2,9 \mathrm{~g} / \mathrm{dL}$ de lipídios e CALIL \& FALCÃO (2003) citam ainda que os valores de lipídios no leite de colostro podem variar de 1,8 a 2,9 g/dL.

Os processos de congelamento e descongelamento, podem acarretar rompimento das membranas dos glóbulos de gordura, propiciando a coalescência e facilitando a aderência às paredes dos frascos (VIEIRA et al., 2004), assim o teor de gordura e o valor energético podem ter apresentado valores menores, provavelmente devido à lipólise do leite humano. Ao comparar as concentrações encontradas com o proposto por CALIL \& FALCÃO (2003) observa-se que nas amostras estudadas o valor de gordura esta muito superior ao limite aceitável.

As concentrações de proteínas, açúcares e minerais analisadas estão detalhadas na Tabela 3. O LH possui uma composição nutricional balanceada e sofre mudanças de acordo com o estágio de lactação (STEVANATO et al., 2012). Fatores associados aos maus hábitos da mãe podem contribuir também para uma mudança da composição nutricional deste leite.

TABELA 3: Concentração de proteínas totais, açúcares e minerais nas amostras de LH de mães dependentes químicas (Crack).

\begin{tabular}{lcc}
\hline & L $_{1}$ & $\mathrm{LH}_{2}$ \\
\hline Proteínas Totais $(\mathrm{g} / \mathrm{L})$ & 12,7 & 13,1 \\
Lactose $(\mathrm{g} / \mathrm{dL})$ & 14,72 & 25,90 \\
Glicose $(\mathrm{mg} / \mathrm{dL})$ & 36,47 & 37,65 \\
Sacarose $(\mathrm{g} / \mathrm{dL})$ & 10,89 & 11,24 \\
$\mathrm{Na}(\mathrm{mg} / \mathrm{dL})$ & 21,24 & 29,56 \\
$\mathrm{~K}(\mathrm{mg} / \mathrm{dL})$ & 4,76 & 10,2 \\
$\mathrm{Ca}(\mathrm{mg} / \mathrm{dL})$ & 21,57 & 24,63 \\
\hline
\end{tabular}

Os resultados médio encontrados de proteínas totais no leite das duas mães foi de 12,90 $\pm 0,28 \mathrm{~g} / \mathrm{L}$ (Tabela 3). Considerando o leite analisado sendo o colostro, por ter sido coletado nos dias próximos ao nascimento da criança, CARMO (2003) enfatiza que a concentração de proteína que um leite de colostro deve ter aproximadamente 15,8 g/L, já CURY (2009) cita que o leite de colostro deve conter aproximadamente $23 \mathrm{~g} / \mathrm{L}$ de proteínas. Ao comparar essas referências com os resultados encontrados neste experimento, observou-se um valor inferior ao proposto por CARMO (2003) e CURY (2009), no leite das duas mães analisadas.

Essa diminuição é vista como ruim, pois CALIL \& FALCÃO (2003) enfatizam que é importante que a quantidade de proteínas esteja adequada porque o recémnascido e o lactente são dotados de uma atividade anabólica intensa, dificilmente igualada em qualquer outro período de suas vidas; tal atividade necessita, pois, de uma oferta correspondente de nitrogênio a ser fornecida nos primeiros seis meses de vida.

CALIL \& FALCÃO (2003) citam ainda, que a composição de proteínas do leite inclui a alfa-lactalbumina, a lactoferrina, a lisozima, a soroalbumina, as imunoglobulinas e a betalactoglobulina. A alfa-lactalbumina, que constitui cerca de $40 \%$ das proteínas do soro do LH, é necessária para o transporte de ferro e ainda para a síntese de lactose na glândula mamária. Assim como a lactoferrina, a lisozima e as imunoglobulinas, especialmente a $\operatorname{lgA}$ secretória, são proteínas do soro do leite humano envolvidas no sistema de proteção. 
Para a análise de lactose, a concentração média determinada no leite das duas mães dependentes químicas foi de 20,31 $\pm 7,90 \mathrm{~g} / \mathrm{dL}$ (Tabela 3). Esses dados demonstram uma concentração aumentada, quando comparada à literatura na qual CURY (2009), enfatiza que a concentração média de lactose em leite de colostro é de $5,7 \mathrm{~g} / \mathrm{dL}$.

Segundo CURY (2003), a taxa de síntese de lactose pela glândula é muito sensível à quantidade de alimento consumido e de acordo com CALIL \& FALCÃO (2003) a alimentação precoce estimula a atividade endógena da lactase (dissacaridase que faz hidrólise da lactose transformando-a em glicose e galactose). Ainda de acordo com CALIL \& FALCÃO (2003), a lactose é responsável por fornecer ao redor de 45 a $50 \%$ do conteúdo energético total do leite humano. Sendo assim, o LH analisado neste trabalho, que foi encontrado uma quantidade aumentada de lactose, não vai fornecer os fatores benéficos ao recém-nascido, como um leite com quantidades adequadas de lactose forneceria.

A quantidade de açúcares totais está representada na Tabela 3 através da concentração de glicose e sacarose presentes no LH das duas mães dependes química. Para a glicose o valor médio encontrado foi de $37,06 \pm 0,83 \mathrm{mg} / \mathrm{dL}$ e para a sacarose $11,07 \pm 0,25 \mathrm{~g} / \mathrm{dL}$.

Em um estudo realizado em mães não dependentes químicas (STEVANATO Et al., 2012), a análise da concentração de sacarose resultou em uma concentração média de 2,56 g/dL para o LH no primeiro mês de lactação, comparando esse dado com a concentração encontrada para o LH de mães dependentes químicas, pode-se observar um grande aumento na concentração de sacarose. Comportamento semelhante foi observado para a concentração de glicose no $\mathrm{LH}$, uma vez que no estudo obtido por STEVANATO et al., (2012) a concentração média foi de 25,67 $\mathrm{mg} / \mathrm{dL}$ para o primeiro mês de lactação, valor este inferior ao determinado neste trabalho $(37,06 \mathrm{mg} / \mathrm{dL})$.

O leite materno sofre influencia de muitas variáveis quanto a sua composição nutricional (LEVY \& BÉRTOLO, 2012). Essas variáveis se baseiam em idade materna, paridade, saúde, classe social, idade gestacional e principalmente estágio de lactação (SALDIVA 2011). De acordo com MORGANO (2005), o leite de colostro é o leite que apresenta níveis mais elevados de minerais, seguido do leite de transição e leite maduro.

A Tabela 3 mostra os valores das concentrações de $\mathrm{Na}, \mathrm{K}$ e Ca obtidos para o LH. A concentração média para as duas mães usuárias de crack foram: $\mathrm{Na}=25,4$ $\pm 5,89 \mathrm{mg} / \mathrm{dL}, \mathrm{K}=7,5 \pm 2,34 \mathrm{mg} / \mathrm{dL}$ e $\mathrm{Ca}=23,1 \pm 1,75 \mathrm{mg} / \mathrm{dL}$. No estudo realizado por STEVANATO et al., (2012), para o LH no primeiro mês de lactação as concentrações foram: $\mathrm{Na}=13,79 \mathrm{mg} / \mathrm{dL}, \mathrm{K}=9,11 \mathrm{mg} / \mathrm{dL}$ e $\mathrm{Ca}=26 \mathrm{mg} / \mathrm{dL}$.

O valor proposto por CALIL \& FALCÃO (2003), é de que a concentração de $\mathrm{Na}$ no LH, oscile ao redor de 16,09 mg/dL. De acordo com CURY (2009) o LH deve conter $\sim 74 \mathrm{mg} / \mathrm{dL}$ de $\mathrm{K}$, o que comparado à concentração encontrada no leite das mães analisadas pode-se observar uma diminuição bastante significativa, pois a concentração encontrada apresenta-se em torno de dez (10) vezes menor do que o ideal, estabelecido por CURY (2009). A concentração de cálcio no LH deve ser de $23 \mathrm{mg} / \mathrm{dL}$ no primeiro mês de lactação (CURY, 2009). Comparando os dados obtidos neste trabalho com os dados da literatura (CURY, 2009; STEVANATO et al., 2012) não tem alteração significativa de valor.

Segundo CARMO et al., (2003), a composição de $\mathrm{Na}, \mathrm{K}$ e Ca não são influenciadas pela dieta materna, sendo que, variações podem ocorrer na lactação ao longo do dia e durante uma mesma mamada, havendo diferenças nas ENCICLOPÉDIA BIOSFERA, Centro Científico Conhecer - Goiânia, v.13 n.24; p. 13812016 
concentrações do leite no início ou no fim da amamentação. Porém, CALIL \& FALCÃO (2003) enfatizam que a concentração de cálcio pode ser influenciada pelo conteúdo de citrato e de caseína, uma vez que o Ca encontrar-se no LH sob a forma ionizada e assim podendo estar acoplado ao fosfato das micelas dessas proteínas.

\section{CONCLUSÃO}

$\mathrm{O}$ LH de mães dependentes químicas (Crack) apresentou diferenças significativas em sua composição nutricional quando comparados com o LH de mães não dependentes. As concentrações de proteína, glicose e os minerais $\mathrm{Na}$ e $\mathrm{K}$ encontraram-se abaixo do estabelecido, entretanto, a acidez, teor de creme, lactose e sacarose encontraram-se acima dos valores estipulados na literatura. $\mathrm{Na}$ análise de gorduras apesar de não ter sido encontrada uma concentração ideal na literatura no leite de mães não dependentes, não houve uma alteração na concentração tão significativa quanto o encontrado nos outros componentes.

É importante ressaltar também a escassez de estudos complementares relacionados ao tema, sugerimos também novas pesquisas com o intuito de colaborar com a comunidade científica, promovendo a saúde de mães e bebês através da divulgação de resultados inéditos, a fim de mobilizar a sociedade e profissionais da saúde acerca do tema. Destacamos que a composição nutricional do LH sofre influência de diversos fatores, como por exemplo: idade materna, paridade, saúde, classe social, idade gestacional, estágio de lactação, individualidade genética bem como de outros elementos que compõe o LH.

\section{REFERÊNCIAS}

AMERICAN, Academy of Pediatrics Committee on Drugs. Transfer of drugs and other chemicals into human milk. Pediatrics; 108(3):776-89, 2001. Disponível em: <doi: 10.1542/peds.108.3.776>;

CALIL, V.M.L.T.; FALCÃO, M.C.; Composição do leite materno: os alimentos ideais. Revista de Medicina, jan-dez. v.82 n.1-4, p. 1-10, 2003. Disponível em: < http://dx.doi.org/10.11606/issn.1679-9836.v82i1-4p1-10>

CARMO, M. G. T.; COLARES, L.G.T.; SAUNDERS, C. Nutrição na Lactação. In: ACCIOLY, Elizabeth; SAUNDERS, Claudia; LACERDA, Elisa Maria de Aquino. Nutrição em obstetrícia e pediatria. Rio de Janeiro: Cultura Médica, cap. 10. p. 225-286, 2003.

CASATTI, G.F.S.; Projeto de intervenção social com gestantes e/ou puérperas, usuárias de drogas lícitas e/ou ilícitas. Ensaios e Ciência: Ciências Biológicas, Agrárias e da Saúde, v. 15, no, 1, p. 97-120, 2011. Disponível em < http://sare.anhanguera.com/index.php/rensc/article/viewArticle/2849>;

CAVALCANTE, J. L. P. TELLES, F. J. S.; PEIXOTO, M. M. L. V.; RODRIGUES, R. C. B. Uso da Acidez Titulável no Controle de Qualidade do Leite Humano Ordenhado. Ciênc. Tecnol. Aliment., Campinas, v.25, n.1, p. 103-107, jan. - mar. 2005. Disponível em: < http://www.scielo.br/pdf/\%0D/cta/v25n1/a16v25n1.pdf.>

CROUCH, J. B.; RUBIN, L. P. Nutrição. In: CLOHERTY, John P.; STARK, Ann R. Manual de neonatologia. Rio de Janeiro: Medsi, cap. 30. p. 618-635, 1993. 
CURY, T. F. Aleitamento Materno. In: ACCIOLY, Elizabeth; SAUNDERS, Claudia; LACERDA, Elisa Maria de Aquino. Nutrição em obstetrícia e pediatria. Rio de Janeiro: Cultura Médica, cap. 15. p. 287-301, 2003.

CURY, T. F. Aleitamento Materno. In: ACCIOLY, Elizabeth; SAUNDERS, Claudia; LACERDA, Elisa Maria de Aquino. Nutrição em obstetrícia e pediatria. Rio de Janeiro: Cultura Médica, cap. 18. p. 279-295, 2009.

FLEET, A.I.R.; LINZELL, J.L. A rapid method of estimating fat in vary small quantities of milk. J. Physiol., v. 175, p. 15P-17P, 1964.

GASPARIN, M.; SILVEIRA, J. L.; GARCEZ, L. W.; LEVY, B. S. Comportamento motor oral e global de recém-nascidos de mães usuárias de crack e/ou cocaína. Rev Sociedade Brasileira de fonoaudiologia, v.17, n. 4, p.459-63, 2012. Disponível em: <http://www.scielo.br/pdf/rsbf/v17n4/16.pdf>

LEVY, L.; BÉRTOLO, H.; Manual do aleitamento materno. Comitê português para a UNICEF. ed. $2012 . \quad$ Disponível em: $<$ https://www.unicef.pt/docs/manual_aleitamento_2012.pdf>

LUCAS, A.; GIBBS, J.A.H.; LYSTER, R.L.J.; BAUN, J.D. Creamatocrit: simple clinical technique for estimating fat concentration and energy value of human milk. British. Med. J., v. 1, p. 1018-1020, 1978. Disponível em: <doi: http://dx.doi.org/10.1136/bmj.1.6119.1018>

MORGANO, M. A.; SOUZA, L. A.; NETO, J. M.; RONDÓ, P. H. C. Composição mineral do leite materno de bancos de leite. Ciênc. Tecnol. Aliment, v. 25 n. 4, p.819-824, 2005. Disponível em: < www.scielo.br/pdf/cta/v25n4/27657.pdf>

PULCHERIO, G. STOLF, A. R.; PETTENON, M.; FENSTERSEIFER, D. P.; KESSLER, F. Crack - da pedra ao tratamento. Revista da AMRIGS, Porto Alegre, v.54, no. 18, p. 337-343, jul.-set. 2010. Disponível em: <www.amrigs.org.br/revista/54-03/018-610_crack_NOVO.pdf>

SALDIVA, S. R.; VENANCIO, S. I.; GOUVEIA, A. G. C.; CASTRO, A. L. S.; ESCUDER, M. M. L.; GIUGLIANI, E. R. J. Influência regional no consumo precoce de alimentos diferentes do leite materno em menores de seis meses residentes nas capitais brasileiras e Distrito Federal. Caderno de Saúde Pública, v. 27, p. 2253-62, 2011. Disponível em:

https://www.researchgate.net/profile/Silvia Saldiva/publication/51833531_Regional i nfluence_on_early_consumption_of_foods_other_than_breast_milk_in_infants_less than_6_months_of_age_in_Brazilian_State_capitals_and_the_Federal_District/links/ $544 \overline{4 f} 6 \overline{7} \mathrm{~b} 0 \mathrm{cf} 2534 \mathrm{c} \overline{7} 65 \mathrm{fea} 4 \overline{3}$.pdf>

SÃO PAULO - Instituto Adolfo Lutz. Métodos físico-químicos para análise de alimentos. Organizadores: Odair Zenebon, Neus Sadocco Pascuet e Paulo Tiglea. São Paulo: Instituto Adolfo Lutz, 1020p. 2008 
SILVA, R. C.; ESCOBEDO, J. P. GIOIELLI, L. A.; QUINTAL, V. S.; IBIDI, S. M.; ALBUQUERQUE, E. M. Composição Centesimal do Leite Humano e Caracterização das Propriedades Fisico-Químicas de sua Gordura. Quimica Nova, v. 30, n. 7, p. 1535-1538, 2007.

SILVA, R. N.; MONTEIRO, V. N.; ALCANFOR, J. D. X.; ASSIS, E. M.; ASQUIERI, E. R. Comparação de métodos para a determinação de açúcares redutores e totais em mel. Ciênc. Tecnol. Aliment, set-dez. v. 23 no 3, p. 337-341, 2003.

STEVANATO, J. O.; GONÇALVES, R. A. C.; OLIVEIRA, A. J. B; GONÇALVES, J.E. Avaliação da Composição Química do Leite Materno. In: VELHO, A. P. M. \& MASSUDA, E. M. Promoção da Saúde. $1^{\text {a }}$ Edição. Maringá: Cesumar, p. 115 - 132, 2012

VIEIRA, A. A.; MOREIRA, M. E. L.; ROCHA, A. D.; PIMENTA, H. P.; LUCENA, S. L. Análise do conteúdo energético do leite humano administrado a recém-nascidos de muito baixo peso ao nascimento. Jornal de Pediatria, v. 80 p. 490 - 494, 2004.

VELOSO, A. C. A.; TEIXEIRA, N.; FERREIRA, I . M; FERREIRA, M. A. Metodologias de doseamento das proteínas do leite. Boletim de Biotecnologia, 2001. 